PROCEEDINGS OF THE

AMERICAN MATHEMATICAL SOCIETY

Volume 126, Number 9, September 1998, Pages 2619-2622

S 0002-9939(98)04409-8

\title{
THE SUPREMUM OF THE DIFFERENCE BETWEEN THE BIG AND LITTLE FINITISTIC DIMENSIONS IS INFINITE
}

\author{
SVERRE O. SMAL $\varnothing$
}

(Communicated by Ken Goodearl)

\begin{abstract}
For each natural number $n$, an example of a finite dimensional algebra $\Lambda_{n}$ is given, which has left little finitistic dimension equal to 1 and left big finitistic dimension equal to $n$.
\end{abstract}

\section{INTRODUCTION}

The objective of this note is to give, for each natural number $n \geq 1$, an example of a finite dimensional algebra $\Lambda_{n}$ over a field such that the left little finitistic dimension of $\Lambda_{n}$ is 1 and the left big finitistic dimension of $\Lambda_{n}$ is $n$.

Recall that the left little finitistic dimension of an algebra $\Lambda$ is the supremum of the projective dimensions of the finitely generated left $\Lambda$-modules of finite projective dimension, and that the left big finitistic dimension of an algebra $\Lambda$ is the supremum of the projective dimensions of all $\Lambda$-modules having finite projective dimension. The first example of a finite dimensional algebra $\Lambda$ where the two dimensions do not coincide was given in 1992 by Birge Huisgen-Zimmermann [1]. The examples given there are a family of monomial relation algebras, and they are rather complicated compared to the algebras given in this note. In [1] it was also proved that, for monomial relation algebras, the difference between the two dimensions is at most 1 , and in the examples the left little finitistic dimension was at least 2. For monomial relation algebras it was also proved that the two dimensions have to coincide if $\mathfrak{r}^{3}=0$ where $\mathfrak{r}$ denotes the radical of the algebra. However, the algebras given in this note have the property that $\mathfrak{r}^{3}=0$.

An alternative approach to the problem of making the difference between the little and big finitistic dimensions arbitrarily large, which was pointed out to me by Jeremy Rickard, is to use the first example constructed by Birge HuisgenZimmermann together with properties of tensor products of algebras. For finite dimensional algebras $\Lambda_{1}$ and $\Lambda_{2}$ over an algebraically closed field $k$ one has that the global dimension of $\Lambda_{1} \otimes \Lambda_{2}$ is the sum of the global dimension of $\Lambda_{1}$ and the global dimension of $\Lambda_{2}$. Letting $\ell$ fin. $\operatorname{dim} \Lambda$ denote the left little finitistic dimension of $\Lambda$ and $\ell$ Fin. $\operatorname{dim} \Lambda$ denote the left big finitistic dimension of $\Lambda$ one also gets that $\ell$ fin. $\operatorname{dim} \Lambda_{1} \otimes \Lambda_{2}=\ell$ fin. $\operatorname{dim} \Lambda_{1}+\ell$ fin. $\operatorname{dim} \Lambda_{2}$ and $\ell$ Fin. $\operatorname{dim} \Lambda_{1} \otimes \Lambda_{2}=$ $\ell$ Fin. $\operatorname{dim} \Lambda_{1}+\ell$ Fin. $\operatorname{dim} \Lambda_{2}$. From these two equalities one can just take repeated

Received by the editors May 22, 1996 and, in revised form, February 24, 1997.

1991 Mathematics Subject Classification. Primary 16E10, 16G10, 16G20,16P10.

Supported by the Norwegian Research Council and by the U.S.-Norway Fulbright Foundation.

(C)1998 American Mathematical Society 
tensor products of the algebra mentioned above to obtain arbitrary differences between the two finitistic dimensions. I include the arguments presented to me by Rickard that the two equalities above hold. First observe that if $M$ is a $\Lambda_{1}$-module of projective dimension $m$ and $N$ is a $\Lambda_{2}$-module of projective dimension $n$, then $M \otimes N$ has projective dimension $m+n$ as a $\Lambda_{1} \otimes \Lambda_{2}$-module. Hence the inequalities $\ell$ fin. $\operatorname{dim} \Lambda_{1} \otimes \Lambda_{2} \geq \ell$ fin. $\operatorname{dim} \Lambda_{1}+\ell$ fin. $\operatorname{dim} \Lambda_{2}$ and $\ell$ Fin. $\operatorname{dim} \Lambda_{1} \otimes \Lambda_{2} \geq$ $\ell$ Fin. $\operatorname{dim} \Lambda_{1}+\ell$ Fin. $\operatorname{dim} \Lambda_{2}$ follow readily. We now carry through the argument for the other inequality for the little finitistic dimension. If one lets $X$ be a finite dimensional $\Lambda_{1} \otimes \Lambda_{2}$-module of finite projective dimension, then $X$ as a $\Lambda_{1}$ module has finite projective dimension, since a $\Lambda_{1} \otimes \Lambda_{2}$-projective resolution restricts to a $\Lambda_{1}$-projective resolution. Hence the $m$-th syzygy, $\Omega^{m} X$, in the $\Lambda_{1} \otimes \Lambda_{2^{-}}$ projective resolution of $X$ is projective as a $\Lambda_{1}$-module when $m=\ell$ fin. $\operatorname{dim} \Lambda_{1}$. Next observe that if $P$ is a projective $\Lambda_{1} \otimes \Lambda_{2}$-module then the isomorphism $\left(P \otimes_{\Lambda_{1}} M\right) \otimes_{\Lambda_{2}} N \simeq P \otimes_{\Lambda_{1} \otimes \Lambda_{2}}(M \otimes N)$ shows that $P \otimes_{\Lambda_{1}} M$ is a flat and hence projective $\Lambda_{2}$-module for all $\Lambda_{1}$-modules $M$. Now let $S$ be a simple $\Lambda_{1}$-module. Then $\Omega^{m} X \otimes_{\Lambda_{1}} S$ has finite projective dimension as a $\Lambda_{2}$-module since a $\Lambda_{2}$-projective resolution can be obtained by tensoring the $\Lambda_{1} \otimes \Lambda_{2}$-resolution of $\Omega^{m} X$ by $S$ over $\Lambda_{1}$ and observing that this sequence is exact since $\Omega^{m} X$ is projective as a $\Lambda_{1}$-module. Now using the isomorphism $\operatorname{Tor}_{i}^{\Lambda_{2}}\left(\Omega^{m}(X) \otimes_{\Lambda_{1}} S, T\right) \simeq \operatorname{Tor}_{i}^{\Lambda_{1} \otimes \Lambda_{2}}\left(\Omega^{m}(X), S \otimes T\right)$ for any simple $\Lambda_{2}$-module $T$, and letting $n$ be the finitistic dimension of $\Lambda_{2}$, we obtain that $\operatorname{Tor}_{i}^{\Lambda_{1} \otimes \Lambda_{2}}(X, S \otimes T)=0$ for all $i>m+n$. Hence the flat dimension and therefore also the projective dimension of $X$ are at most $m+n$ as a $\Lambda_{1} \otimes \Lambda_{2}$-module.

Observe that using the tensor product construction one also increases the Loewy lengths of the algebras involved.

One can now ask if the two equalities involving the finitistic dimensions are valid also for nonseparable finite dimensional algebras over a field.

For a historical account of the finitistic dimension conjectures, we refer to the paper [2].

This paper was written while the author was visiting The University of California at Santa Barbara and he would like to take the opportunity to thank the algebra group there for their hospitality.

\section{The EXAMPLE}

The algebra $\Lambda_{n}$ will be given as a path algebra of a quiver modulo relations and it will then be explained how it is obtained by means of repeated one point extensions using basically injective modules except in the first step. This point of view will then serve as basis for the argument leading to the announced conclusion.

Let $\Gamma_{n}$ be the quiver

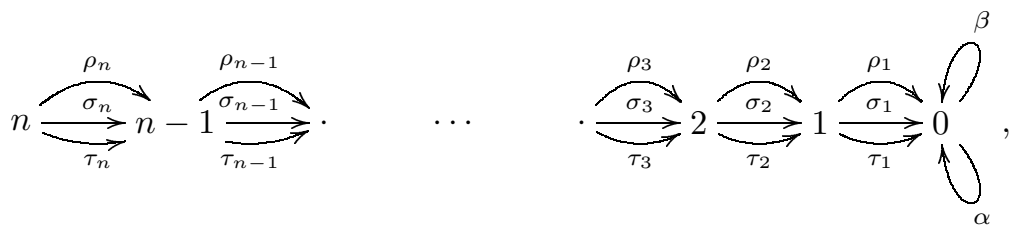

let $k$ be any field, and let $\Lambda$ be the path algebra of $\Gamma$ over $k$ modulo the ideal generated by the following relations: $\alpha^{2}, \beta^{2}, \alpha \beta, \beta \alpha, \alpha \rho_{1}, \alpha \sigma_{1}, \beta \tau_{1}, x_{i} y_{i+1}$ for $i=$ $1,2, \cdots, n-1$ and $x \neq y ; x, y \in\{\rho, \sigma, \tau\}$ and $x_{i} x_{i+1}-y_{i} y_{i+1}, x, y \in\{\rho, \sigma, \tau\}, i=$ $1, \cdots, n-1$. 
Theorem. For each natural number $n \geq 1$, the algebra $\Lambda_{n}$ given above has left little finitistic dimension 1 and left big finitistic dimension $n$.

Proof. For each vertex $i=1,2, \cdots, n$ in the quiver $\Gamma_{n}$, let $e_{i}$ be the corresponding idempotent in $\Lambda_{n}$, and let $P_{i}=\Lambda e_{i}$ and $S_{i}=P_{i} / \mathfrak{r} P_{i}$ be representatives of the indecomposable projective and simple $\Lambda_{n}$-modules respectively. Here $\mathfrak{r}$ denotes the radical of the algebra $\Lambda_{n}$. Since the Loewy length of $P_{0}$ is 2 and all the indecomposable projective $\Lambda_{n}$-modules except $P_{0}$ have Loewy length 3 , there can be no inclusion from a projective $P$ into the radical of another projective $Q$ if $P$ contains an indecomposable direct summand not isomorphic to $P_{0}$. So, in order to have an inclusion of a nonzero projective module $P$ into the radical of another projective module $Q$, the projective $P$ has to be isomorphic to a direct sum of copies of $P_{0}$. However, $P_{0}$ has nonzero morphisms only to $P_{1}, P_{2}$ and itself. So if $P$ is a finitely generated projective module which is a submodule of the radical of a finitely generated projective module, we have the situation $f: P_{0}^{m} \rightarrow P_{0}^{m_{0}} \amalg P_{1}^{m_{1}} \amalg P_{2}^{m_{2}}$ where the image of $f$ is in the radical. But then, since the image of $p_{0} f$, where $p_{0}$ is the projection of $P_{0}^{m_{0}} \amalg P_{1}^{m_{1}} \amalg P_{2}^{m_{2}}$ onto $P_{0}^{m_{0}}$, and the image of $p_{2} f$, where $p_{2}$ is the projection of $P_{0}^{m_{0}} \amalg P_{1}^{m_{1}} \amalg P_{2}^{m_{2}}$ onto $P_{2}^{m_{2}}$, are both semisimple, we obtain an inclusion $P_{0}^{m} \rightarrow P_{1}^{m_{1}}$ by composing the given inclusion $f$ with the projection $p_{1}$ of $P_{0}^{m_{0}} \amalg P_{1}^{m_{1}} \amalg P_{2}^{m_{2}}$ onto $P_{1}^{m_{1}}$. However, it is not hard to see that this is possible only if $m_{1} \geq m$. But then $\mathfrak{r}^{2} P_{1}^{m_{1}}$, which has dimension $3 m_{1}$ as a $k$-vector space, is not contained in the image of $P_{0}^{m}$ which has an intersection with $r^{2} P_{1}^{m_{1}}$ of dimension $2 m$. Therefore the cokernel of any inclusion $P \rightarrow Q$ of finitely generated projective $\Lambda_{n}$-modules $P$ and $Q$ with the image in the radical has Loewy length three and can therefore not be embedded in the radical of any projective $\Lambda_{n}$-module. Therefore the syzygy of any finitely generated $\Lambda_{n}$-module cannot have projective dimension 1 , which shows that the left little finitistic projective dimension of $\Lambda_{n}$ is 1 for all $n \geq 1$.

Next, let $e_{0, i}$ be the coordinates of $P_{0}^{(\mathbb{N})}$ with $e_{0}$ in the $i$ 'th place and 0 otherwise, and let analogously $e_{1, i}$ be the coordinates of $P_{1}^{(\mathbb{N})}$ with $e_{1}$ in the $i$ th place and 0 otherwise. Letting $\phi: P_{0}^{(\mathbb{N})} \rightarrow P_{1}^{(\mathbb{N})}$ be given by $\phi\left(e_{0,2 i-1}\right)=\tau_{1} e_{1,2 i-1}+\sigma_{1} e_{1, i}$ and $\phi\left(e_{0,2 i}\right)=\tau_{1} e_{1,2 i}+\rho_{1} e_{1, i}$, where $\tau_{1}, \sigma_{1}$ and $\rho_{1}$ also denote the cosets of $\tau_{1}, \sigma_{1}$ and $\rho_{1}$ in $\Lambda_{n}$ respectively, it is not hard to verify that $\phi$ is an inclusion $P_{0}^{(\mathbb{N})} \rightarrow P_{1}^{(\mathbb{N})}$ such that coker $\phi$ is annihilated by the residue classes of $\alpha$ and $\beta$ and has socle equal to $S_{0}^{(\mathbb{N})}$. If we now go back and look at the definition of the algebra $\Lambda_{n}$, it follows that for $n \geq 3$ one has that $\Lambda_{n}$ is a one point extension of $\Lambda_{n-1}$ by the injective envelope of $S_{n-2}$ considered as a $\Lambda_{n-1}$-module. $\Lambda_{2}$ is the one point extension of $\Lambda_{1}$ by the injective $\Lambda_{1} /(\alpha, \beta)$-envelope of $S_{0}$ considered as a $\Lambda_{1}$-module, where we also let $\alpha$ and $\beta$ denote the residue classes of $\alpha$ and $\beta$ in $\Lambda_{1}$. Denoting coker $\phi$ by $X_{1}$, we know that we can embed $X_{1}$ into $P_{2}^{(\mathbb{N})}$ in such a way that their socles coincide, yielding a quotient module which we denote by $X_{2}$ having Loewy length two with socle isomorphic to $S_{1}^{(\mathbb{N})}$. This can now be repeated leading to an exact sequence of modules

$$
0 \rightarrow P_{0}^{(\mathbb{N})} \rightarrow P_{1}^{(\mathbb{N})} \rightarrow P_{2}^{(\mathbb{N})} \rightarrow \cdots \rightarrow P_{n}^{(\mathbb{N})} \rightarrow X_{n} \rightarrow 0
$$

where each $P_{i}^{(\mathbb{N})}$ is projective. This shows that the big finitistic dimension of $\Lambda_{n}$ is at least $n$. 
That the big finitistic dimension cannot exceed $n$ follows from the fact that the vertices in $\Gamma_{n}$ corresponding to all indecomposable projective $\Lambda_{n}$-modules $P_{i}$ except $P_{0}$ do not belong to any oriented cycle in $\Gamma_{n}$. This finishes the proof of the theorem.

\section{REFERENCES}

1. B. Zimmermann Huisgen, Homological domino effect and the first finitistic dimension conjecture, Invent. Math, 108 (1992), 369-383. MR 93i:16016

2. B. Zimmermann Huisgen, The Finitistic Dimension Conjectures-A Tale of 3.5 Decades, in; Abelian Groups and Modules, Proceedings of the Padova Conference 1994, A. Facchini and C. Menini, eds., Kluwer 1995. MR 97a:16018

Department of Mathematics, Lade, The Norwegian University for Science and TechNOLOGY, 7034 TrondheIM, NORWAY

E-mail address: sverresm@math.ntnu.no 